\title{
Efeitos da sinvastatina na mucosite gastrointestinal induzida por 5-fluorouracil em ratos.
}

\section{Effects of simvastatin on 5-fluorouracil-induced gastrointestinal mucositis in rats.}

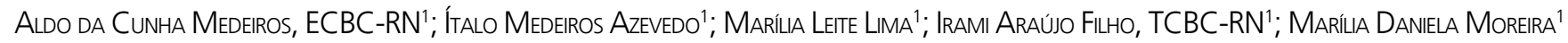

R E S U M O

Objetivo: examinar os efeitos da sinvastatina na mucosite gástrica e intestinal após o tratamento com 5-fluorouracil (5FU), determinados pela expressão de citocinas e histologia em ratos. Métodos: ratos pesando $270 \pm 15 \mathrm{~g}$ foram divididos em dois grupos. O grupo 5-FU+salina foi tratado com 5-FU $(50 \mathrm{mg} / \mathrm{kg})$ mais solução salina a 0,9\% por gavagem uma vez ao dia por cinco dias. O grupo 5-FU+sinvastatina foi tratado com 5-FU (50mg/kg), mais sinvastatina (10mg/kg), da mesma forma. Foi feita a eutanásia dos animais no sexto dia. O estômago e o intestino foram fotografados e removidos para exame. Dosagens séricas de TNF-a, IL-1B, IL-6 e histopatologia (coloração HE) do estômago e intestino foram realizadas. Resultados: o peso corporal diminuiu em ratos no grupo 5-FU+salina. A sinvastatina não inibiu a perda de peso induzida pelo 5-FU. Danos significativos da mucosa no estômago e no jejuno foram observados em ratos que receberam apenas 5-FU. As dosagens séricas de citocinas foram significativamente menores no grupo 5-FU+sinvastatina do que no grupo 5-FU $(p<0,05)$. A sinvastatina causou efeitos protetores significativos contra as lesões da mucosa gástrica e jejunal induzidas por 5-FU. Conclusão: a sinvastatina atenua a mucosite gástrica e intestinal relacionada à terapêutica com 5-FU. Nossos dados encorajam futuros estudos pré-clínicos e clínicos sobre a utilidade das estatinas na prevenção da mucosite gastrointestinal.

Descritores: Mucosite. Quimioterapia Adjuvante. Sinvastatina. Roedores.

\section{INTRODUÇÃO}

A mucosite oral e gastrointestinal induzida por quimioterapia é um dos eventos adversos mais comuns da quimioterapia para o câncer ${ }^{1}$. Está associada a um alto risco de infecção, dor, redução da dose de quimioterapia e morte relacionada à infecção. A diretriz de prática clínica para a prevenção e tratamento da mucosite gastrointestinal induzida pela quimioterapia do câncer foi publicada em $2004^{2}$. Sonis et al. descreveram as perspectivas sobre a lesão da mucosa induzida pela terapia do câncer ${ }^{3}$.

Foram relatadas alterações quantitativas e qualitativas da mucina gastrointestinal em animais experimentais tratados com 5-fluorouracil (5-FU), demonstrando sua importância na barreira da mucosa gastrointestinal ${ }^{4}$. A mucosite gastrointestinal induzida durante o tratamento do câncer é considerada um efeito colateral grave da quimioterapia; promove ulceração grave e inflamação do trato gastrointestinal, principalmente no intestino delgado ${ }^{5,6}$. A interrupção do tratamento do câncer devido à mucosite geralmente leva a uma redução nas taxas de cura, aumenta os custos do tratamento, diminui a qualidade de vida e, consequentemente, agrava o prognóstico da doença ${ }^{7}$. Atualmente, não há intervenção curativa para a mucosite inflamatória induzida pelo tratamento do câncer. Medidas paliativas para a mucosite na clínica incluem crioterapia oral, aplicação de laser suave e administração sistêmica de medicamentos, como glicocorticoides, fatores de crescimento, e outros ${ }^{8,9}$. Considerando o alto custo de alguns procedimentos terapêuticos para mucosite e sua baixa eficácia, tem sido considerado o desenvolvimento de tratamentos alternativos, estatinas e produtos naturais ${ }^{10}$.

Alguns dos efeitos benéficos das estatinas, independentes do colesterol ou "pleiotrópicos", incluem a melhora da função endotelial pela regulação positiva da óxido nítrico sintase endotelial (eNOS), proliferação de macrófagos $^{10}$, redução da atividade

1 - Universidade Federal do Rio Grande do Norte, Departamento de Cirurgia, Natal, RN, Brasil. 
plaquetária ${ }^{11}$, estabilização de placas ateroscleróticas ${ }^{12}$, propriedades antioxidantes ${ }^{13}$, efeitos anti-inflamatórios e imunomoduladores ${ }^{14}$. Dada a importância do assunto, o presente estudo tem como objetivo analisar os efeitos da sinvastatina em um modelo experimental de mucosite gastrointestinal induzida por 5-FU, determinados por citocinas e marcadores histológicos.

\section{MÉTODOS}

O protocolo experimental foi aprovado pelo Comitê de Ética em Pesquisa do Hospital Universitário Onofre Lopes-UFRN/CEUA (no 07/17). Os animais foram tratados de acordo com os princípios estipulados pela Lei 11.794/2008 para o manuseio e manutenção de animais de laboratório.

\section{Animais}

Utilizamos ratos Wistar (Rattus norvegicus albinus, Rodentia mammalia) pesando $270 \pm 18 \mathrm{~g}$ neste estudo. Esses animais eram do Centro de Ciências da Saúde da UFRN-Brasil. Eles foram alojados em gaiolas individuais em um ambiente controlado por temperatura e umidade, com um ciclo de luz escura de 12 horas. No início do período experimental, os animais foram pesados após jejum de 24h. Durante o tratamento abaixo mencionado, os ratos receberam alimentos (Prevence®) e água ad libitum.

\section{Concepção do experimento e procedimentos}

Os animais foram divididos aleatoriamente em dois grupos de seis ratos cada. Para todos os ratos, o 5-FU foi administrado oralmente por sonda gástrica $(50 \mathrm{mg} / \mathrm{kg})$ uma vez por dia, durante cinco dias. No grupo experimental (5-FU/SIMV - n=6), a suspensão oral de sinvastatina $(10 \mathrm{mg} / \mathrm{kg})$ foi administrada por gavagem, começando no primeiro dia da administração de 5-FU, por cinco dias. No grupo (5-FU/SAL - $n=6$ ), solução salina $0,9 \%$ foi administrada nas mesmas doses e limites de tempo. Os animais foram pesados diariamente para controle de peso.
Após cinco dias de tratamento com 5-FU, o sangue dos animais foi coletado por punção cardíaca sob anestesia com ketamina $70 \mathrm{mg} / \mathrm{kg}$ e xilazina $10 \mathrm{mg} / \mathrm{kg}$ por via intraperitoneal (ip) e os ratos foram mortos com overdose de tiopental sódico (100mg/ $\mathrm{kg}$ ) ip. O sangue foi centrifugado e o soro separado para dosagem de TNF- $\alpha$, IL-1ß e IL- 6 pela técnica ELISA, utilizando kits Peprotec, EUA, de acordo com as instruções do fabricante.

Após tricotomia e antissepsia da parede abdominal, foi realizada uma laparotomia mediana para documentação fotográfica da cavidade. O estômago e o intestino delgado foram então removidos para exame macroscópico e microscópico.

\section{Exame macroscópico do estômago e do intestino delgado (jejuno)}

O estômago foi aberto com incisão na grande curvatura, e o intestino (jejuno), com incisão longitudinal. Eles foram examinados quanto a edema, ulcerações e hemorragia, de acordo com os critérios: edema intenso (3 pontos), quando líquido era visto externamente e internamente na parede do estômago ou intestino; moderado (2 pontos), quando o edema estava confinado à mucosa; leve (1 ponto), entre normal e moderado; nenhum edema (pontuação $0)$, quando normal. A intensidade da hemorragia foi determinada da seguinte forma: coágulo e sangue intraluminal (3 pontos); hematomas mucosos e de parede (2 pontos); telangiectasia ou dilatação dos vasos (1 ponto); normal (pontuação 0).

\section{Histopatologia}

O estômago e o intestino foram fixados em formalina tamponada a 10\%, embebidos em parafina, cortados a $5 \mu \mathrm{m}$ de espessura e corados com hematoxilina e eosina. O exame histopatológico foi realizado por um patologista sem conhecimento prévio dos grupos estudados, analisando cinco campos microscópicos de cada órgão por animal. 
O material foi classificado de acordo com os escores padrão em uma escala de 0 a 3, de acordo com Lima et al. ${ }^{15}$ : 0 , tecido conjuntivo e epitelial sem vasodilatação, infiltrado celular inflamatório ausente ou discreto, ausência de hemorragia, edema, úlceras ou abscessos; 1, congestão vascular discreta, infiltração celular discreta de leucócitos mononucleares, ausência de hemorragia, edema, úlceras ou abcessos; 2, congestão vascular moderada, degeneração hidrópica epitelial (vacuolização), infiltrado celular moderado com predomínio de leucócitos polimorfonucleares, presença de áreas hemorrágicas, edema e pequenas úlceras ocasionais; 3, congestão vascular aguda, vasodilatação acentuada, infiltrado celular intenso, predominantemente polimorfonuclear, presença de áreas hemorrágicas, edema e úlceras extensas. Esses critérios foram adaptados para o estômago e intestino, com base no estudo da mucosite oral ${ }^{16}$.

\section{Análise estatística}

Os resultados dos dados macro e microscópicos foram expressos como média \pm desvio padrão, usando o teste $\mathrm{t}$ de Student e o software BioEstat 5.0. Diferenças entre os grupos foram consideradas significativas quando $p<0,05$.

\section{RESULTADOS}

Nos registros de peso corporal, os ratos tratados com 5-FU/salina apresentaram maior perda de massa corporal que os ratos tratados com 5-FU/ sinvastatina $(p<0,05)$.

\section{Dosagem de citocina sérica}

A expressão do TNF- $\alpha$ ocorreu em menor grau no soro de ratos tratados com 5-FU + sinvastatina $(172,6 \pm 18 \mathrm{pg} / \mathrm{ml})$ do que naqueles recebendo $5-\mathrm{FU}+$ soro fisiológico $(347,5 \pm 63 \mathrm{pg} / \mathrm{ml})$.
A expressão de $\mathrm{IL}-1 \mathrm{~b}$ foi significativamente maior no grupo que recebeu solução salina (5-FU/SAL, $122,9 \pm 26 \mathrm{pg} / \mathrm{ml}$ ) do que no grupo sinvastatina (5-FU/SIMV, 44,8 $\pm 8 \mathrm{pg} / \mathrm{ml})$. A expressão de IL-6 também foi menor no grupo 5-FU/SIMV (52,4 $13 \mathrm{pg} / \mathrm{ml})$ do que no grupo 5-FU/SAL $(123,6 \pm 18 \mathrm{pg} / \mathrm{ml})$. Os dados estão resumidos na tabela 1.

Tabela 1. Valores de citocinas séricas de animais submetidos ao tratamento de 5-FU, associado com solução salina ou sinvastatina.

\begin{tabular}{lccc}
\hline \multicolumn{1}{c}{ Grupos } & $\begin{array}{c}\text { TNF- } \alpha \\
(\mathrm{pg} / \mathrm{mL})\end{array}$ & $\begin{array}{c}\mathrm{IL}-1 \beta \\
(\mathrm{pg} / \mathrm{mL})\end{array}$ & $\begin{array}{c}\mathrm{IL}-6 \\
(\mathrm{pg} / \mathrm{mL})\end{array}$ \\
\hline 5-FU/SAL & $347,5 \pm 63$ & $122,9 \pm 26$ & $123,6 \pm 18$ \\
5-FU/SIMV & $172,6 \pm 18^{*}$ & $44,8 \pm 8^{*}$ & $52,4 \pm 13^{*}$ \\
\hline
\end{tabular}

* $p<0,05$ em comparação com as dosagens dos ratos do grupo 5-FU/SAL, teste $t$ de Student; 5-FU/SAL, grupo de ratos tratados com 5-fluorouracil e soro fisiológico; 5-FU/ SIMV, grupo ratos tratados com 5-fluorouracil e sinvastatina.

\section{Histopatologia}

O estômago e jejuno nos órgãos expostos ao 5-FU apresentaram citoesqueleto deformado, aumento de contagem de células necróticas, redução de vilosidades, atrofia, criptas e camada muscular, associado a edema, ruptura de criptas, infiltrado inflamatório e vacuolização. Com o tratamento com sinvastatina, os sinais de citotoxicidade no estômago e no jejuno induzidos pela exposição ao 5-FU tiveram notáveis melhorias morfológicas, bem como, marcados pela redução do número de células necróticas e inflamação. A figura 1-A mostra fotomicrografias da imagem estomacal representativa de animal tratado com sinvastatina, e a 1-B, de um tratado com solução salina. A figura 2-A mostra fotomicrografia do intestino delgado de rato tratado com sinvastatina, e a 2-B, de animal tratado com solução salina. Comparando os escores extraídos das imagens histopatológicas, as diferenças na microscopia foram significativas $(p<0,05)$. Esses dados estão resumidos na tabela 2 . 

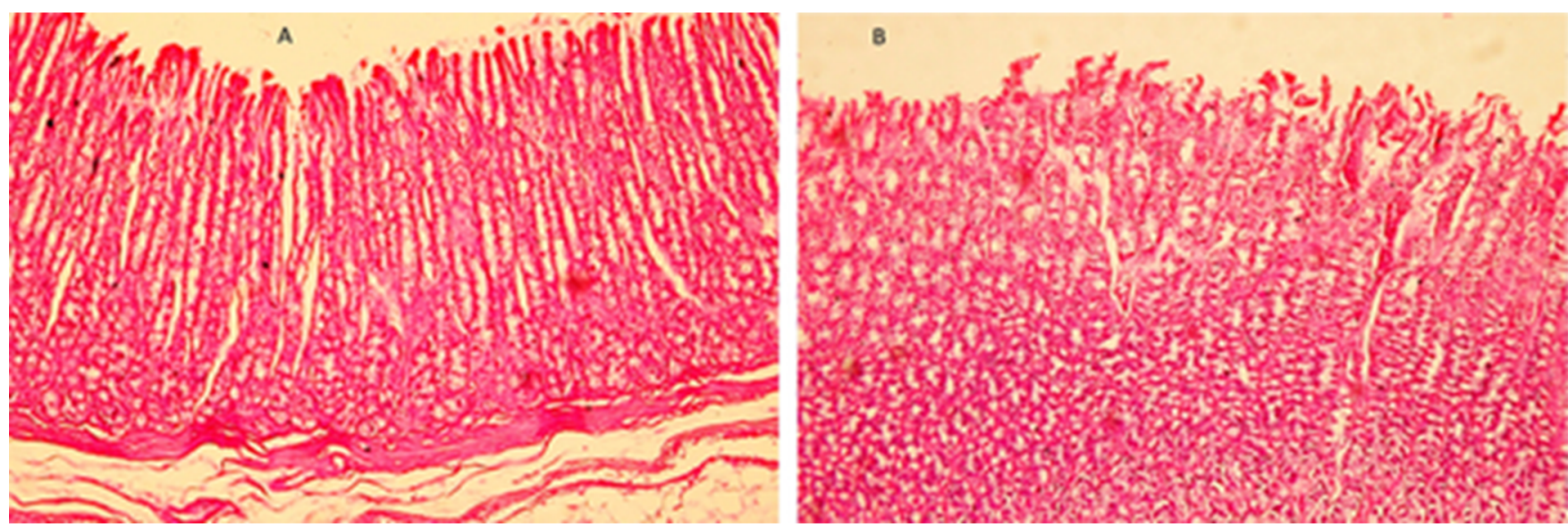

Figura 1. A) fotomicrografia de estômago de animal do grupo 5-FU/sinvastatina; B) estômago de rato do grupo 5-FU/salina. H-E 200x.
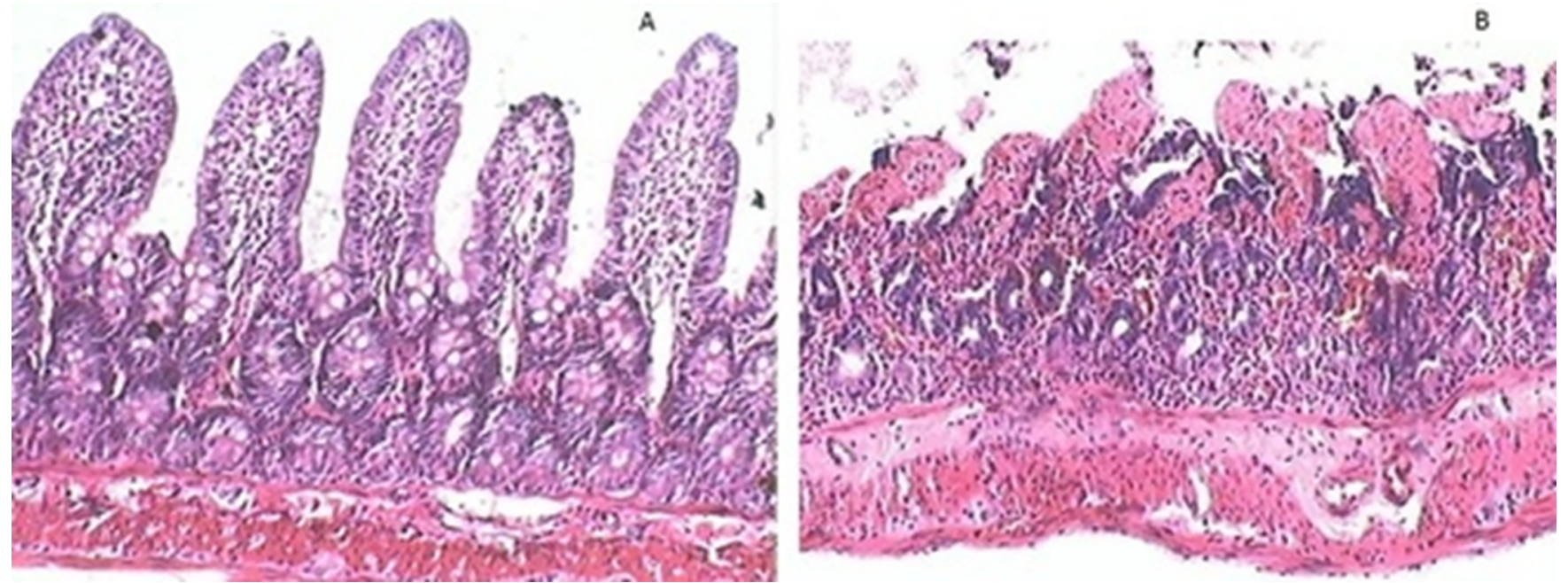

Figura 2. A) fotomicrografia de intestino de animal do grupo 5-FU/sinvastatina; B) intestino de rato do grupo 5-FU/salina. H-E 200x.

Tabela 2. Valores das pontuações histológicas no estômago e jejuno dos animais submetidos ao tratamento com 5-FU, solução salina ou sinvastatina.

\begin{tabular}{lcl}
\hline \multicolumn{1}{c}{ Grupos } & Estômago & Jejuno \\
\hline 5-FU/SAL & $26 \pm 3,2$ & $20 \pm 2,7$ \\
$5-F U / S I M V$ & $14 \pm 1,8^{*}$ & $9 \pm 2,2^{*}$ \\
\hline
\end{tabular}

* $p<0,05$ quando comparadas as potuações histológicas dos órgãos examinados pelo teste $t$ de Student; 5-FU/SAL, grupo ratos tratados com 5-FU e soro fisiológico; 5-FU/ SIMV, grupo ratos tratados com 5-FU e sinvastatina.

A macroscopia revelou sangue e coágulos no interior do estômago e do jejuno, bem como, ulcerações da mucosa gástrica em todos os animais tratados com solução 5-FU + salina (Figura 3). Estas alterações foram observadas apenas em um rato do grupo 5-FU + sinvastatina. Nos outros, a mucosa estava edemaciada, sem sangue e ulcerações.

\section{DISCUSSÃO}

O 5-FU continua sendo um dos quimioterápicos mais utilizados no tratamento do câncer gástrico avançado devido à sua extensa atividade antitumoral, bem como, ao sinergismo com outros fármacos antineoplásicos ${ }^{17}$. No entanto, a sua eficácia é relativamente baixa, devido à alta frequência das suas toxicidades hematológica e gastrointestinal. A mucosite gastrointestinal é um efeito colateral comum induzido pelo 5-FU, levando a interrupções e atrasos no tratamento de tumores, um dos principais determinantes da morbidade e mortalidade na oncologia ${ }^{18}$. Apesar das tentativas de reduzir a incidência e a intensidade da mucosite, atualmente não há tratamento profilático e/ou terapêutico efetivo². 

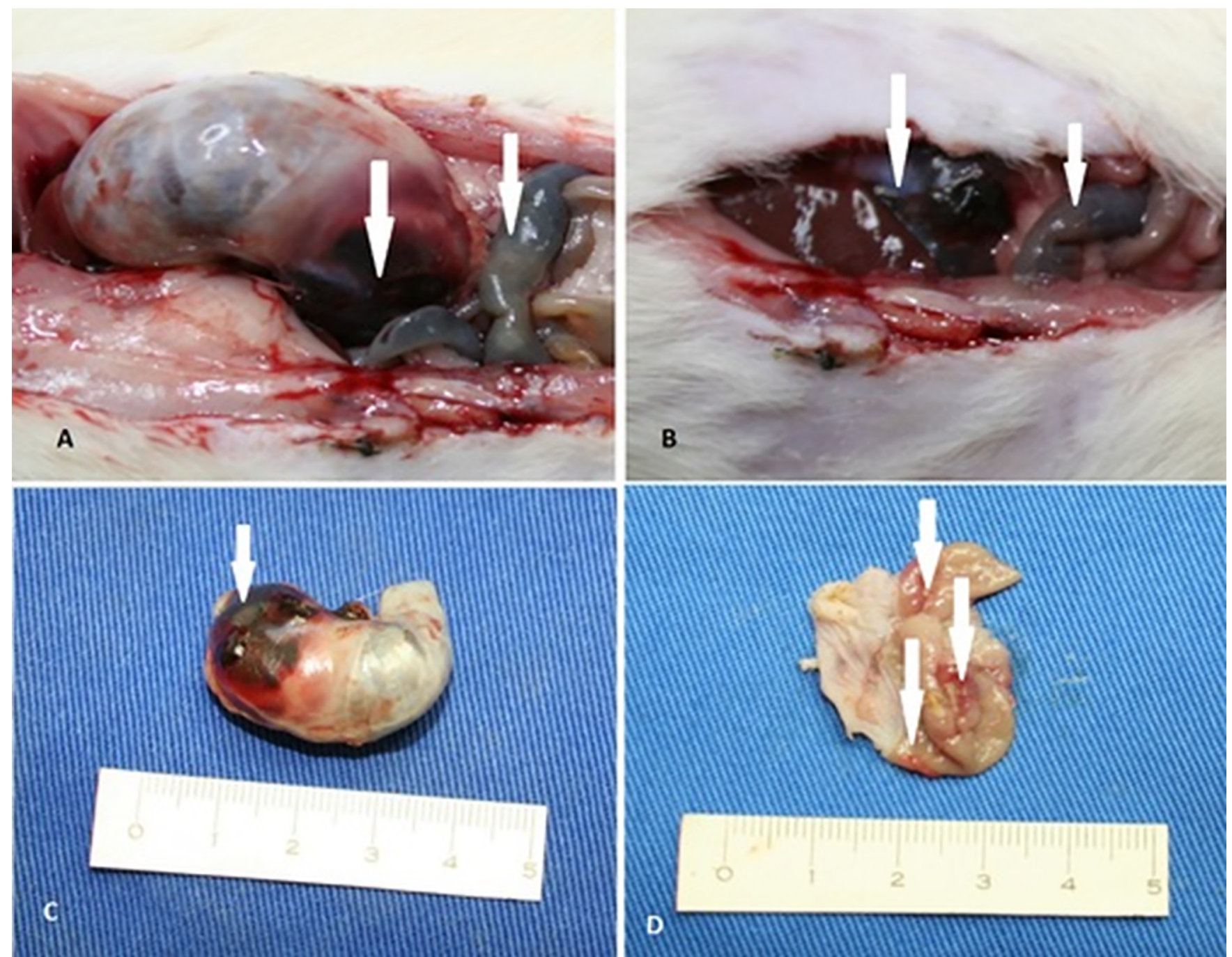

Figura 3. A) abdome de um rato do grupo 5-FU/salina, aberto por laparotomia. Estômago e intestino (setas) cheios de sangue; B) intestino cheio de sangue (setas); C) sangue visto pela transparência no estômago do rato (seta); D) estômago aberto, mostrando úlceras mucosas (setas).

Alguns estudos demonstraram um papel protetor das estatinas contra a citotoxicidade induzida pela radioterapia e doxorubicina em células endoteliais in vitro ${ }^{19}$, bem como, contra citotoxicidade e respostas inflamatórias in vivo ${ }^{20,21}$. Estes dados apontam para um papel organoprotetor mais geral das estatinas contra o dano tecidual causado por drogas antineoplásicas e radioterapia. Assumindo que as células endoteliais são de particular relevância para as respostas inflamatórias, a protecção das células endoteliais pelas estatinas é indicativa da sua atividade antimucosite no tratamento com 5-FU in vivo. Esta hipótese corrobora dados que mostram que as estatinas previnem danos à mucosa e inflamação em hamsters após administração de 5-FU22.
No entanto, os anti-inflamatórios não hormonais não atenuaram a mucosite oral induzida por radiação no modelo do camundongo ${ }^{23}$, mostrando que opções preventivas e terapêuticas alternativas são necessárias. Nossos resultados demonstraram que a sinvastatina exerceu efeitos significativos na proteção da mucosa gástrica e intestinal em ratos tratados com 5-FU. Os mecanismos subjacentes ao desenvolvimento desta mucosite foram investigados; uma hipótese relatada é que a via da ciclo-oxigenase exerce papel na lesão e na dor tecidual por meio da suprarregulação da prostaglandina E2 que evoca a dor e das citocinas pró-inflamatórias ${ }^{24,25}$. De fato, nossos resultados 
demonstraram que os níveis séricos de TNF- $\alpha$, IL$1 \mathrm{~B}$ e IL- 6 estavam elevados em ratos tratados com 5-FU, e o tratamento com sinvastatina reduziu significativamente a expressão dessas citocinas pró-inflamatórias. Um estudo anterior do nosso laboratório que examinou a cistite induzida pela ciclofosfamida concluiu que o pré-tratamento com sinvastatina atenuou a inflamação do urotélio e diminuiu a atividade das citocinas ${ }^{26}$.

Zeigler et al. observaram efeitos inibitórios da lovastatina em queratinócitos humanos in vitro, após tratamento com dois tipos diferentes de mucosite induzida por quimioterapia antineoplásica. Além disso, verificou-se que a estatina é genoprotetora nos queratinócitos e também revelou atividade citoprotetora, relacionada à inibição da morte celular por apoptose ${ }^{27}$.
Nossos resultados in vivo sugerem que a sinvastatina pode ser útil para a profilaxia e tratamento da mucosite gastrointestinal, em vez de melhorar o repovoamento do epitélio danificado, que é uma estratégia terapêutica preferida. Nossos dados pré-clínicos fornecem uma base promissora para futuros estudos clínicos que abordam a questão de se estatinas, isoladamente ou em combinação com drogas que estimulam processos regenerativos, podem melhorar as medidas de suporte com o objetivo de aliviar a mucosite induzida por quimioterapia.

Em conclusão, a sinvastatina atenua a mucosite gástrica e intestinal relacionada à terapêutica com 5-FU em modelo animal. Estes dados encorajam futuros estudos clínicos e in vivo abordando a utilidade das estatinas na prevenção da mucosite gastrointestinal.

\title{
A B S T R A C T
}

\begin{abstract}
Objective: simvastatin has pleiotropic anti-inflammatory and immunomodulatory effects potentially usefull to prevent chemotherapy-induced gastrointestinal mucositis. Studies on this are scarce. This study aimed to examine the effects of simvastatin on gastric and intestinal mucositis after 5-fluorouracil (5-FU) treatment in rats. Methods: rats weighing $270 \pm 18 \mathrm{~g}$ were divided into two groups. The 5-FU+saline group (5-FU/SAL) rats were treated with 5-FU (50mg/kg) plus $0.9 \%$ saline orally (gavage) once daily for five days. The 5-FU+simvastatin (5-FU/SIMV) group was treated with 5-FU $(50 \mathrm{mg} / \mathrm{kg})$, plus simvastatin $(10 \mathrm{mg} / \mathrm{kg})$, in the same way. The rats were euthanased on the sixth day, then their stomach and intestine were photographed and removed for exams. Dosages of serum TNF-a, IL-1B, IL-6 and histopathology were done for stomach and intestine. Results: body-weight was significantly lower in rats treated with 5-FU+saline than the weight loss of the 5-FU/SIMV group rats. TNF-a expression was lower in 5-FU/SIMV group (172.6 $\pm 18 p g / m l)$ than in 5-FU/ SAL (347.5 $\pm 63 \mathrm{pg} / \mathrm{ml})$. Serum IL-1 b was lower in 5-FU/SAL group $(134.5 \pm 23 \mathrm{pg} / \mathrm{ml})$ than in 5-FU/SIMV (48.3 $\pm 9 \mathrm{pg} / \mathrm{ml})$. Serum IL-6 was 61.8 $\pm 15 \mathrm{pg} / \mathrm{ml}$ in 5-FU/SIMV and $129.4 \pm 17 \mathrm{pg} / \mathrm{ml}$ in 5-FU/SAL groups. These differences were significant (p<0.05). Mucosal damage in stomach and jejunum were observed in rats receiving 5-FU alone. In the stomach and jejunum, simvastatin caused significant protective effects against 5-FU-induced mucosal injury. Conclusion: simvastatin attenuated gastric and intestinal mucositis related to 5-FU therapeutics in animal model. These data encourage forthcoming clinical studies addressing the usefulness of statins in the prevention and treatment of gastrointestinal mucositis.
\end{abstract}

Keywords: Chemotherapy, Adjuvant. Mucositis. Simvastatin. Rodentia.

\section{REFERÊNCIAS}

1. Keefe DM, Schubert MM, Elting LS, Sonis ST, Epstein $\mathrm{JB}$, Raber-Durlacher JE, Migliorati CA, McGuire DB, Hutchins RD, Peterson DE; Mucositis Study Section of the Multinational Association of Supportive Care in Cancer and the International Society for Oral Oncology. Updated clinical practice guidelines for the prevention and treatment of mucositis. Cancer. 2007;109(5):820-31.
2. Rubenstein $E B$, Peterson $D E$, Schubert $M$, Keefe $D$, McGuire D, Epstein J, Elting LS, Fox PC, Cooksley C, Sonis ST; Mucositis Study Section of the Multinational Association for Supportive Care in Cancer; International Society for Oral Oncology. Clinical practice guidelines for the prevention and treatment of cancer therapy-induced oral and gastrointestinal mucositis. Cancer. 2004;100/9 Suppl):2026-46. 
3. Sonis ST, Elting LS, Keefe D, Peterson DE, Schubert M, Hauer-Jensen M, Bekele BN, Raber-Durlacher J, Donnelly JP, Rubenstein EB; Mucositis Study Section of the Multinational Association for Supportive Care in Cancer; International Society for Oral Oncology. Perspectives on cancer therapy-induced mucosal injury: pathogenesis, measurement, epidemiology, and consequences for patients. Cancer 2004;100/9 Suppl):1995-2025.

4. Saegusa Y, Ichikawa T, Iwai T, Goso Y, Okayasu I, Ikezawa $T$, et al. Changes in the mucus barrier of the rat during 5-fluorouracil-induced gastrointestinal mucositis. Scand J Gastroenterol. 2008;43(1):5965.

5. Yeoh AS, Gibson RJ, Yeoh EE, Bowen JM, Stringer AM, Giam KA, et al. A novel animal model to investigate fractionated radiotherapy-induced alimentary mucositis: the role of apoptosis, p53, nuclear factor-kappaB, COX-1, and COX-2. Mol Cancer Ther. 2007;6(5):2319-27.

6. Han X, Wu Z, Di J, Pan Y, Zhang H, Du Y, et al. CXCL9 attenuated chemotherapy-induced intestinal mucositis by inhibiting proliferation and reducing apoptosis. Biomed Pharmacother. 2011;65(8):54754.

7. Touchefeu $\mathrm{Y}$, Montassier $\mathrm{E}$, Nieman $\mathrm{K}$, Gastinne T, Potel G, Bruley des Varannes S, et al. Systematic review: the role of the gut microbiota in chemotherapy- or radiation-induced gastrointestinal mucositis - current evidence and potential clinical applications. Aliment Pharmacol Ther. 2014;40(5):409-21.

8. Keefe DM. Gastrointestinal mucositis: a new biological model. Support Care Cancer. 2004;12(1):6-9.

9. Lalla RV, Schubert MM, Bensadoun RJ, Keefe D. Anti-inflammatory agents in the management of alimentary mucositis. Support Care Cancer. 2006;14(6):558-65.

10. Aikawa $M$, Rabkin $E$, Sugiyama $S$, Voglic SJ, Fukumoto $Y$, Furukawa $Y$, et al. An HMG-CoA reductase inhibitor, cerivastatin, suppresses growth of macrophages expressing matrix metalloproteinases and tissue factor in vivo and in vitro. Circulation. 2001;103(2):276-83.
11. Huhle G, Abletshauser C, Mayer N, Weidinger G, Harenberg J, Heene DL. Reduction of platelet activity markers in type II hypercholesterolemic patients by a HMG-CoA-reductase inhibitor. Thromb Res. 1999;95(5):229-34.

12. Rosenson RS, Brown AS. Statin use in acute coronary syndromes: cellular mechanisms and clinical evidence. Curr Opin Lipidol. 2002;13(6):625-30.

13. Stoll LL, McCormick ML, Denning GM, Weintraub NL. Antioxidant effects of statins. Drugs Today (Barc). 2004;40(12):975-90.

14. Shin SK, Cho JH, Kim EJ, Kim EK, Park DK, Kwon KA, et al. Anti-inflammatory and anti-apoptotic effects of rosuvastatin by regulation of oxidative stress in a dextran sulfate sodium-induced colitis model. World J Gastroenterol. 2017;23(25):4559-68.

15. Lima V, Brito $G A$, Cunha FQ, Rebouças CG, Falcão BA, Augusto RF, et al. Effects of the tumor necrosis fator-alpha inhibitors pentoxifyline and thalidomide in short-term experimental oral mucositis in hamsters. Eur J Oral Sci. 2005;113(3):210-7.

16. Wanzeler AMV, Júnior SMA, Gomes JT, Gouveia EHH, Henriques HYB, Chaves RH, et al. Therapeutic effect of andiroba oil (Carapa guianensis Aubl.) against oral mucositis: an experimental study in golden Syrian hamsters. Clin Oral Investig. 2017;22(5):2069-79.

17. Wöhrer SS, Raderer $M$, Hejna $M$. Palliative chemotherapy for advanced gastric cancer. Ann Oncol. 2004;15(11):1585-95.

18. Rolston KV. The burdens of cancer therapy: clinical and economic outcomes of chemotherapy-induced mucositis. Cancer. 2004;100(6):1324-5; author reply 1325-6.

19. Damrot J, Nübel $T$, Epe $B$, Roos WP, Kaina B, Fritz $G$. Lovastatin protects human endothelial cells from the genotoxic and cytotoxic effects of the anticancer drugs doxorubicin and etoposide. Br J Pharmacol. 2006;149(8):988-97.

20. Haydont V, Bourgier C, Pocard M, Lusinchi A, Aigueperse J, Mathé $D$, et al. Pravastatin inhibits the Rho/CCN2/extracellular matrix cascade in human fibrosis explants and improves radiationinduced intestinal fibrosis in rats. Clin Cancer Res. 2007;13(18 Pt1):5331-40. 
21. Henninger C, Huelsenbeck J, Huelsenbeck S, Grösch S, Schad A, Lackner KJ, et al. The lipid lowering drug lovastatin protects against doxorubicin induced hepatotoxicity. Toxicol Appl Pharmacol. 2012;261(1):66-73.

22. Medeiros CA, Leitão RF, Macedo RN, Barboza DR, Gomes AS, Nogueira NA, et al. Effect of atorvastatin on 5-fluorouracil-induced experimental oral mucositis. Cancer Chemother Pharmacol. 2011;67(5):1085-100.

23. Haagen J, Krohn H, Röllig S, Schmidt M, Wolfram K, Dörr W. Effect of selective inhibitors of inflammation on oral mucositis: preclinical studies. Radiother Oncol. 2009;92(3):472-6.

24. Lalla RV, Pilbeam CC, Walsh SJ, Sonis ST, Keefe DM, Peterson DE. Role of the cyclooxygenase pathway in chemotherapy-induced oral mucositis: a pilot study. Support Care Cancer. 2010;18(1):95-103.

25. Morales-Rojas T, Viera N, Morón-Medina A, Alvarez CJ, Alvarez A. Proinflammatory cytokines during the initial phase of oral mucositis in patients with acute lymphoblastic leukaemia. Int J Paediatr Dent. 2012;22(3):191-6.
26. Dantas AC, Batista-Júnior FF, Macedo LF, Mendes MN, Azevedo IM, Medeiros AC. Protective effect of simvastatin in the cyclophosphamideinduced hemorrhagic cystitis in rats. Acta Cir Bras. 2010;25(1):43-6.

27. Ziegler V, Albers A, Fritz G. Lovastatin protects keratinocytes from DNA damage-related proapoptotic stress responses stimulated by anticancer therapeutics. Biochim Biophys Acta. 2016;1863(6 Pt A):1082-92.

Recebido em: 03/02/2018

Aceito para publicação em: 28/08/2018

Conflito de interesse: nenhum.

Fonte de financiamento: CNPq (Conselho Nacional de Desenvolvimento Científico e Tecnológico).

\section{Endereço para correspondência:}

Aldo da Cunha Medeiros

E-mail: aldom@uol.com.br aldo@ufrnet.br

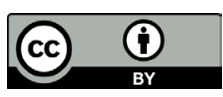

